\title{
Residual stress measurements on a metal matrix composite using the contour method with brittle fracture
}

\author{
Jeferson Araujo de Oliveira' ${ }^{1,{ }^{*}}$, Michael Edward Fitzpatrick ${ }^{1, b}$, Jan Kowal1, c
}

${ }^{1}$ Engineering \& Innovation, The Open University, Milton Keynes, MK7 6AA, UK

ajeferson.oliveira@open.ac.uk, bm.e.fitzpatrick@open.ac.uk, cjan.kowal@open.ac.uk

Keywords: Brittle fracture surface method, fatigue crack, contour method, neutron diffraction.

\begin{abstract}
In this work we evaluate the application of the contour method to fatigue and fracture surfaces. Residual stress measurements were made on quenched and aged $\mathrm{AA} 2124-\mathrm{SiC}_{\mathrm{p}}$ composite using neutron diffraction, the contour method with wire EDM, and the contour method on a fatigue crack surface including brittle failure. The contour method successfully measured residual stresses from a wire electro-discharge cut surface, but the fracture method results suggest that residual stress information is lost due to plasticity during fatigue crack growth.
\end{abstract}

\section{Introduction}

Aluminium-based composites reinforced with $\mathrm{SiC}$ particles $\left(\mathrm{SiC}_{\mathrm{p}}\right)$ have been studied for industrial applications owing to their high specific strength and stiffness, excellent wear resistance and good damage tolerance. The measurement of macro residual stresses (type I RS) [1] on AA2124-SiC $\mathrm{p}_{\mathrm{p}}$ composites is an important step in their characterization because, owing to the heattreatable nature of their aluminium alloy matrix, quenching is usually required to reach optimum performance [2]. However quench residual stresses can lead to distortions or even premature failure of components made of such composites. These residual stresses can be measured by nondestructive techniques, such as neutron diffraction [3] and synchrotron X-ray diffraction, as well as destructive techniques, such as hole drilling and the contour method.

Neutron diffraction (ND) is capable of determining residual stresses non-destructively by measuring residual strains at large depths [4]. In time-of-flight instruments, different wavelengths of a pulsed "white" beam of neutrons incident on the residual stressed sample diffract in different grain families and reach the neutron detectors at different times and angles in relation to the incident beam, which correlate to the lattice parameters of these grains based on Bragg`s law. By comparing these lattice distances with ones measured in a stress-free sample of the same material, residual strains are determined, which can be used for the calculation of residual stresses [1].

The contour method is a residual stress measurement technique capable of determining a 2-D map of residual stresses after a cut. The deformation due to the residual stress release caused by the cut is extracted from measurements of the cut surface contour, and input into a finite element (FE) model in order to calculate a 2-D map of the component of stress normal to that surface [5].

The recently-presented fracture surface method shares the contour method concepts, but this time, instead of a cut, the residual stresses are relaxed by brittle fracture. This method is unique, as residual stress information can be retrieved after the failure of a component [6].

In this work we evaluate the application of the fracture surface method to fatigue and fracture surfaces. Residual stress measurements were made on quenched and aged AA2124 matrix reinforced with silicon carbide particles $\left(\mathrm{SiC}_{\mathrm{p}}\right)$ using the fracture surface method on a fatigue crack surface including brittle failure. For comparison, RS measurements were also made in the same specimen using neutron diffraction and the contour method with wire electro-discharge machining. 


\section{Materials}

Residual stress measurements were performed on a fractured four-point bending fatigue sample. The sample was cut from a hot isostatic pressed and forged MMC plate composed of 2124 aluminium alloy reinforced with $20 \%$ wt. (17\%vol.) $3 \mu \mathrm{m}$ silicon carbide particles manufactured by Materion Aerospace Metal Composites, Farnborough UK. The parent plate underwent a heat treatment consisting of a one hour soak at $505^{\circ} \mathrm{C}$, followed by cold water quenching, and then ageing at $170^{\circ} \mathrm{C}$ for $48 \mathrm{~h}$.

The sample was extracted from the plate in the T-S orientation, according to ASTM E399 (1990) [7]. It was lightly machined by conventional milling techniques, reducing the amount of residual stresses induced by this process, and then the lateral surfaces were polished to an $R_{\mathrm{a}}$ of $0.10 \mu \mathrm{m}$.

The prepared specimen was then subjected to a fatigue crack growth study [8] in which the crack length was monitored and the load amplitude was automatically controlled in order to grow the crack incrementally at constant stress intensity factor amplitudes $\left(\Delta K_{\mathrm{I}}\right)$ between 6 and $9.2 \mathrm{MPa} \sqrt{\mathrm{m}}$, with $R=0.1$. The applied $K_{\mathrm{I}}$ did not take into account any residual stress. After the crack length reached $8.41 \mathrm{~mm}$, a fracture toughness test was performed resulting in a failure surface comprised of a fatigue crack followed by brittle fracture with shear lips, as shown in Fig. 1.

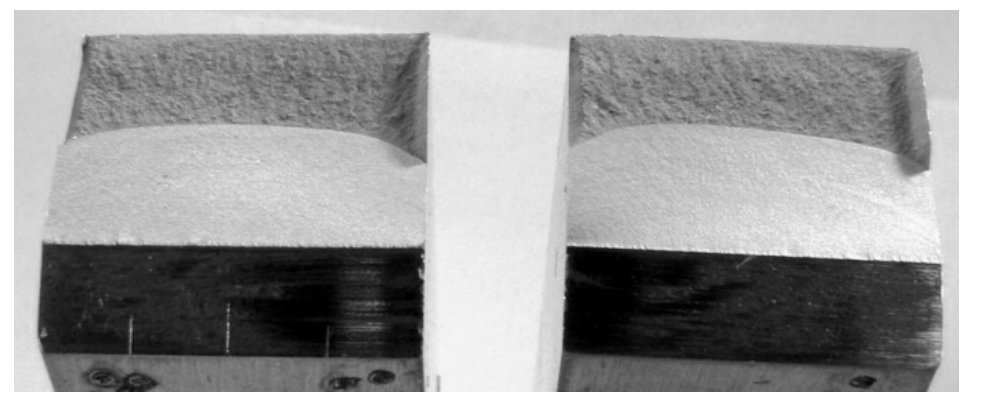

\section{Methods}

Fig. 1: Photograph of the fracture surfaces of the studied sample.

Neutron diffraction. Neutron diffraction measurements were performed at POLDI [9], Paul Scherrer Institut (PSI), Switzerland. The instrument is a time-of-flight diffractometer based on a continuous "white" neutron source where the beam is modulated by a chopper resulting in overlapped neutron pulses. The lattice distances are determined based on the slopes $\mathrm{d} t / \mathrm{d} \theta$ in an arrival time $(t)$ versus scattering angle $(2 \theta)$ contour plot of neutron counts.

The measurements were performed on one half of the fractured specimen sufficiently distant from the fracture and end faces, where it is assumed the effect of the fracture on the RS field is negligible. Two dimensions of the gauge volume, over which the strain values are averaged, were defined by a set of moveable slits that shape the incident beam, while the dimension along the neutron beam travel direction was defined by a radial collimator that only allowed the reflected neutrons coming from a specific section of the beam to reach the detector. The dimensions of gauge volumes were 1.5 by $1.5 \mathrm{~mm}$ on the $x y$ plane, and since along $z$ the strains were not expected to vary significantly, a $20 \mathrm{~mm}$ length was defined in this direction. Fig. 2 illustrates the specimen and the location of the gauge volume. Strain-free references were measured from powders of the matrix alloy and the reinforcement material as present in the composite.

In order to determine the full stress tensor, at least six measurements should be done in each location [4]. However, due to the short time available for the measurements, the principal strain directions were assumed to be aligned with the specimen faces. This is reasonable, given the nature of the expected RS field. Therefore only three principal strain components were measured.

Pawley or Rietveld refinement might lead to high uncertainties due to the low intensities of most measured peaks. Strain profiles were obtained from the analysis of single peak shifts from the lattice planes (311) of the aluminium alloy and (220) of the silicon carbide, assuming that no texture was present in the sample. The (311) lattice plane most closely reflects the macroscopic behaviour of aluminium [10]. 
Each strain profile was then smoothed using quadratic polynomial regression and macrostresses were calculated from them, following the procedure detailed in [3].

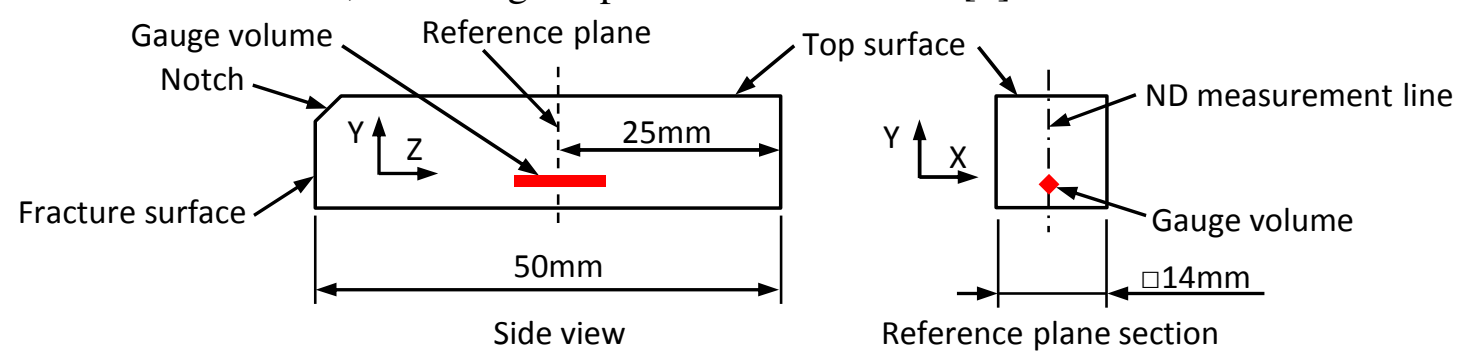

Fig. 2: Schematic with sample dimensions, showing the position of the neutron diffraction strain measurements as well as the reference plane where the contour cut was performed.

Contour method. The contour method was used to measure residual stresses of the specimen in two different places. One measurement was performed using the profiles of the fracture surfaces and the other was the conventional method, i.e. introducing a wEDM cut to release the stresses. The residual stresses in these two locations should be similar, although not directly comparable, because the notch introduced for the fatigue test partially relaxed the normal stresses in the fracture section.

Contour cut. The wEDM machine used for the contour cuts was an Agie Charmilles FI $440 \mathrm{ccS}$. The parameters selected for the contour cuts were a $0.15 \mathrm{~mm}$ diameter uncoated hard brass wire at a cut feed rate of $10 \mathrm{~mm} \cdot \mathrm{min}^{-1}$. The contour cut was performed on the reference plane (see Fig. 2). It is important to note that fracture surface and neutron diffraction measurements were carried out before introducing the contour cut in one fractured half.

Surface measurements and stress calculation. Both the EDM cut and fracture surfaces were measured using a Zeiss Eclipse coordinate measurement machine (CMM) equipped with a TP8 Renishaw touch-trigger probe and an ILD2210 Micro-Epsilon laser triangulation probe. Although the laser system alone can in principle provide a resolution of $0.15 \mu \mathrm{m}$ [11], the resolution of the $\mathrm{CMM}$ is in practice limited to $1 \mu \mathrm{m}$ by the CMM axes. The axes of the CMM are controlled via a PC using Inspect 3D software version 4.00r.

Using the laser sensor, a relatively fine grid of points was measured, at a pitch of $30 \mu \mathrm{m}$ in both $x$ and $y$ directions. In addition, the perimeter of each surface was measured with respect to the same coordinate system, but this time using a $\varnothing 4 \mathrm{~mm}$ ruby tip touch probe. The point spacing for the latter measurement was set to $1 \mathrm{~mm}$ and the perimeters were later used both for filtering out points measured by the laser system outside the perimeter boundary, and creating the model geometry in Abaqus as described later.

After performing the measurements, the points measured on the right half were mirrored about the $y z$ plane. Then the measurements of right and left halves were aligned by translating and rotating the data in the $x y$ plane. Afterwards, the measurement points from both halves were fitted to a common $x y$ grid and finally the out-of-plane coordinates $(z)$ from both halves were averaged to create displacement profiles related to the normal RS $\left(\sigma_{\mathrm{zz}}\right)$ relaxation.

To filter out scatter caused both by the uncertainties on the measurement device and the surface roughness, the displacement profiles were smoothed using bivariate cubic splines with a knot spacing of $3.5 \mathrm{~mm}$ in the $x$ and $y$ directions. This spacing was selected after the analysis of the residuals, i.e. the result of the subtraction of the smoothed from the unsmoothed displacements, as defined in [12].

The negatives of these smoothed displacements were supplied as boundary conditions to an FE model of one cut/fractured half in order to calculate the normal residual stress $\left(\sigma_{\mathrm{zz}}\right)$ formerly present on the cut/fracture surface. For this purpose, Abaqus [13] was used to create 3D isotropic linearelastic models of one half of the sample, based on the perimeter measured by the CMM and in the case of the fracture, including the notch geometry. The elastic constant of the MMC, $100 \mathrm{GPa}$, was supplied and the mesh type used was C3D8R (8-node linear brick with reduced integration and hourglass control). The geometry of the FE models is not presented here, for brevity. The negative of each smoothed displacement was finally applied to the respective model in order to calculate 2D maps of residual stresses $\sigma_{\mathrm{zz}}$. 


\section{Results}

The smoothed residual stress profiles measured by neutron diffraction (see Fig. 3) were consistent with the expected behaviour of a quenched sample and resulted in plausible macrostress trends. However, note that none of the residual stress components equilibrate along the profile, contrary to what would normally be expected in this region of the sample. The balance of the normal component $\left(\sigma_{\mathrm{zz}}\right)$ is positive while that of the remaining components is negative. The possible causes are discussed later.

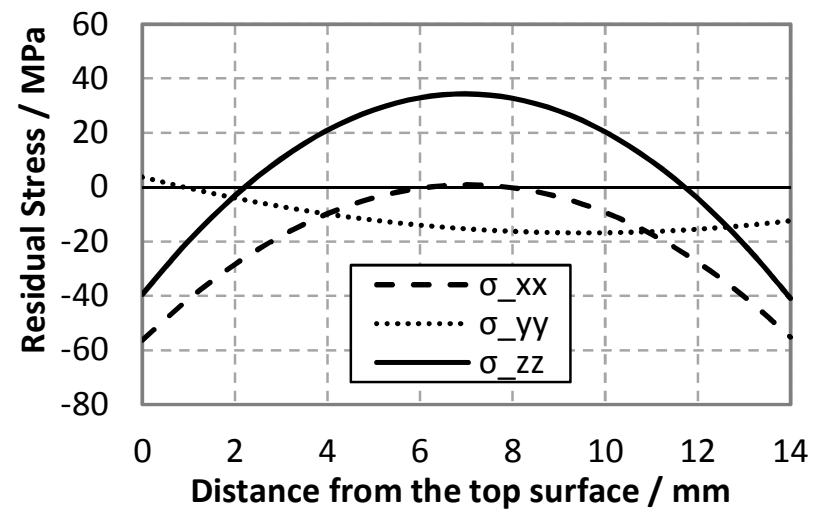

Fig. 3: Smoothed residual stress profiles measured using neutron diffraction.

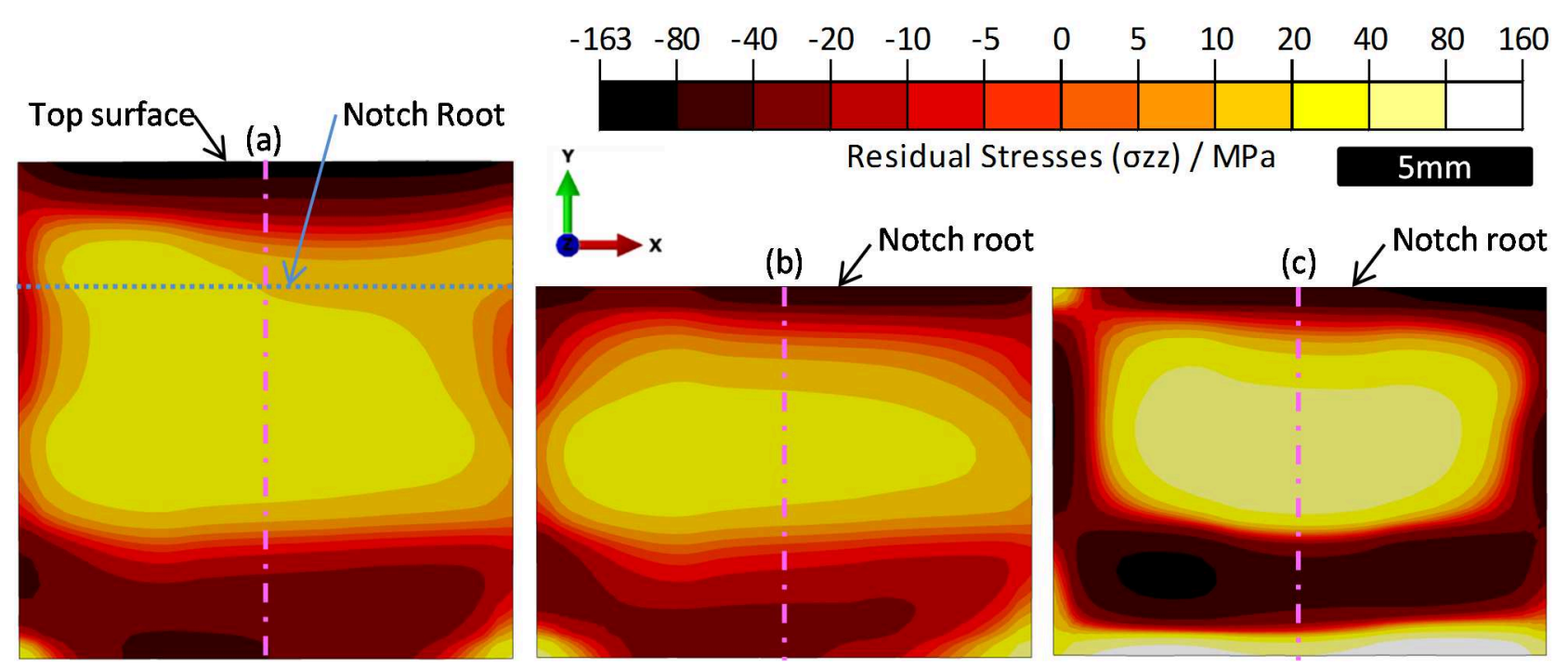

Fig. 4: Residual stress maps $\left(\sigma_{\mathrm{zz}}\right)$ measured (a) via the contour method with a wEDM cut, (b) via the same method forced to balance on part of the cut surface, and (c) via the fracture surface method on a fatigue crack with brittle fracture.

The normal residual stress maps $\left(\sigma_{\mathrm{zz}}\right)$ measured via the contour method on both fracture and cut surfaces are presented as colour-maps on the $x y$ plane (see Figs. 4(a) and (c)). Recall that the presence of the notch makes the fracture section smaller than that of the cut. For this reason, these results cannot be directly compared. Therefore the displacement profile acquired from the cut surface was applied to a new FE model, this time including the cut slot up to the same depth of the notch, forcing RS balance over the same area as the fracture surface, thus direct comparison can be made (see Fig. 4(b)). This approach shares the same assumptions as the contour method.

Fig. 5 presents RS line profiles extracted from the contour cut results along the dash-dotted line in Fig. 4(a) starting from the top surface of the sample for comparison with ND results. Apart from the first millimetre, the contour method results correlate within $20 \mathrm{MPa}$ with the neutron diffraction measurements, which is comparable with the uncertainty of the measurements. In contrast with this good correlation, line profiles from the fatigue with fracture surfaces do not correlate well with contour cut results (Fig. 6). The most probable causes are discussed later. 


\section{Discussion}

The smoothed RS profiles measured by neutron diffraction were according to expectations for a quenched sample and resulted in plausible macrostress trends. However, as noted before, the measured profiles do not equilibrate as expected. This feature could be caused by a combination of errors, including the "stress-free" lattice parameter, the elastic constants, the volume fraction of each phase, any texture possibly present in the sample, and/or the strain uncertainties associated with the strains allied to the small number of points measured. In the case presented, since one RS component shows an overall positive balance and the others show the opposite, the dominant source of error is more likely to be either the high strain uncertainties amplified by the small number of points measured or a possible texture effect, since the other sources would shift all RS components in the same direction. Although the profiles do not balance, the maximum deviation $(18 \mathrm{MPa})$ is less than the overall uncertainty in the measurements.

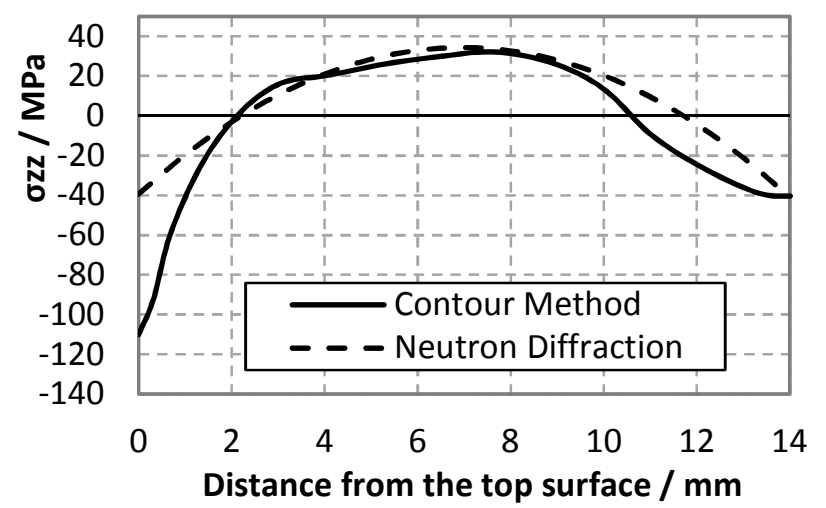

Fig. 5: Residual stress profiles measured on the reference plane via the contour method and neutron diffraction techniques.

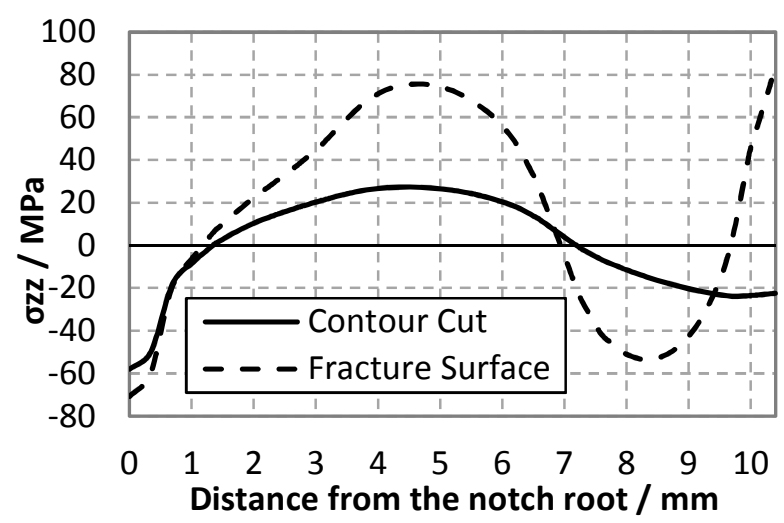

Fig. 6: Residual stress line profiles measured via the contour method with contour cut and fatigue with fracture surface.

Contour results from the wEDM cut showed good results, although a few artefacts can be seen near the edges of the RS profiles in Fig. 4(a). This type of artefact has been studied by Toparli [12], having spline smoothing allied to relatively high uncertainties in the surface measurements as most probable cause. Due to their localized nature, these artefacts do not significantly affect results elsewhere.

Good overall correlation was found between measurements via ND and CM using the cut surface (see Fig. 5). However, apart from the notch root vicinity, fracture surface results using the fatigue crack with brittle fracture depart from the CM even though RS balance was forced over a comparable portion of the cut surface. This suggests that the displacement profiles are significantly affected by the plasticity that occurred during the fatigue crack growth, invalidating the assumption of elastic separation that can be adopted successfully in the case of brittle fractures [6]. 
It is important to note that, in the contour or fracture surface methods, the magnitude of the displacements increases with the specimen size, while the plastic zone size at the fatigue crack tip depend on the material and test parameters. Therefore, for much larger samples, the effect of fatigue plasticity may be negligible, but this is still to be systematically studied.

\section{Conclusions}

1. The contour method is suitable for measuring residual stresses in composites made of AA2124 reinforced with $20 \% \mathrm{wt}$. of $3 \mu \mathrm{m} \mathrm{SiC}$ particles.

2. The fracture surface method is not capable of determining RS maps from a surface comprised of a fatigue crack followed by a brittle fracture, given the size and the stress-strain behaviour of the sample presented in this work.

\section{Acknowledgements}

We wish to thank Materion Aerospace Metal Composites for the provision of the material studied in the project. The authors are grateful for technical support from V. Davydov at PSI, and P. Ledgard and D. Flack at The Open University. JAO and MEF are supported by Lloyd's Register Foundation (LRF), a UK registered charity that helps to protect life and property by supporting engineering-related education, public engagement and the application of research.

\section{References}

[1] P. Withers, Residual stress and its role in failure, Reports on Progress in Physics. 70 (2007) 2211.

[2] M.E. Fitzpatrick, J.E. Moffatt, A. Dubois, F. Gregoire, A.D. Tarrant, Influence of heat treatment processes on fatigue performance of particle reinforced aluminium alloys, Materials Science and Technology. 18 (2002) 1453-1457.

[3] M.E. Fitzpatrick, M.T. Hutchings, P.J. Withers, Separation of macroscopic, elastic mismatch and thermal expansion misfit stresses in metal matrix composite quenched plates from neutron diffraction measurements, Acta Materialia. 45 (1997) 4867-4876.

[4] P.J. Withers, H.K.D.H. Bhadeshia, Residual stress. Part 1 - Measurement techniques, Materials Science and Technology. 17 (2001) 355-365.

[5] M.B. Prime, Cross-sectional mapping of residual stresses by measuring the surface contour after a cut, Journal of Engineering Materials and Technology. 123 (2001) 162-168.

[6] M.B. Prime, A.T. DeWald, M.R. Hill, B. Clausen, M. Tran, Forensic determination of residual stresses and KI from fracture surface mismatch, Engineering Fracture Mechanics (2014), http://dx.doi.org/10.1016/j.engfracmech.2013.12.008.

[7] A.S.T.M. Designation, E399-90. Standard test method for plane-strain fracture toughness of metallic materials, in: 1991 Annual Book of ASTM Standards , pp. 485-51.

[8] M.E. Fitzpatrick, A study of the effects of a quench residual stress field on fatigue in an Al/SiCp metal matrix composite, University of Cambridge, 1995.

[9] U. Stuhr, Time-of-flight diffraction with multiple pulse overlap. Part I: The concept, Nuclear Instruments and Methods in Physics Research A. 545 (2005) 319-329.

[10] B. Clausen, T. Lorentzen, T. Leffers, Self-consistent modelling of the plastic deformation of f.c.c. polycrystals and its implications for diffraction measurements of internal stresses, Acta Materialia. 46 (1998) 3087-3098.

[11] Micro-Epsilon, Instruction manual optoNCDT 22xx, pp. 72.

[12] M.B. Toparli, M.E. Fitzpatrick, S. Gungor, Improvement of the contour method for measurement of near-surface residual stresses from laser peening, Expl Mech. (2013) 1-14.

[13] D.C.S. Simulia, ABAQUS 6.11 analysis user's manual, Abaqus 6.11 Documentation (2011). 